\title{
First case reported of transcatheter aortic valve implantation in a patient affected by Fabry's Disease and severe low-flow low-gradient aortic valve stenosis
}

\author{
Gennaro Giustino, Alaide Chieffo, Matteo Montorfano, Vasileios F. Panoulas, Chiara Bernelli, \\ Pietro Spagnolo, Azeem Latib, Remo Daniel Covello, Ottavio Alfieri, Antonio Colombo \\ Interventional Cardiology Unit, Ospedale San Raffaele, Milan, Italy.
}

Correspondence: Alaide Chieffo. Address: Interventional Cardiology Unit, San Raffaele Scientific Institute, Via Olgettina 6020132 Milan, Italy. Email: chieffo.alaide@hsr.it

Received: February 7, 2014

Accepted: March 16, 2014

Online Published: March 31, 2014

DOI : $10.5430 /$ crim.v1n2p71

URL: http://dx.doi.org/10.5430/crim.v1n2p71

\begin{abstract}
Fabry's disease is a rare X-linked lysosomal storage disorder in which cardiac involvement is common manifesting as arrhythmias, coronary artery disease, valvular disorders and restrictive cardiomyopathy. We describe a case of a 76-year-old female affected by Fabry's disease and severe low-flow low-gradient aortic stenosis that underwent transcatheter aortic valve implantation (TAVI) in our center. No complications occurred during the procedure or in the peri-procedural period. The patient was discharged 5 days later, in a much improved clinical and haemodynamic state. At long-term follow-up there was a remarkable improvement in left ventricular systolic function despite the underlying cardiomyopathy. Despite absence of previous experience, our case report indicates that TAVI can be a feasible and effective therapeutic option for aortic stenosis in patients with Fabry's disease.
\end{abstract}

\section{Keywords}

TAVI, Fabry's disease, Cardiomyopathy, Low-flow low-gradient aortic stenosis

\section{I ntroduction}

Fabry's disease (FD) is a rare X-linked lysosomal storage disorder caused by alpha-galactosidase A enzyme deficiency, resulting in excessive deposition of neutral glycosphingolipid, globotriaosyl-ceramide predominantly in endothelial and vascular smooth muscle cells, causing a multi-systemic disorder affecting cardio-vascular system, kidneys, central and peripheral nervous systems, eyes and skin ${ }^{[1]}$. Cardiac involvement is common and manifests with arrhythmias, coronary artery disease / angina, valvular disorders and restrictive cardiomyopathy with underlying fibrosis ${ }^{[2]}$. Aortic stenosis (AS) or regurgitation, alongside mitral valve disease, are the main clinical presentations of valvular involvement. As demonstrated by histological analyses, valvular disorders are mainly due to accumulation of metabolic substrates within valvular fibroblasts causing leaflet thickening and deformation ${ }^{[3]}$. Considering the systematic multiorgan involvement in this metabolic disorder, these patients often have multiple comorbidities and are therefore deemed at high surgical risk, rendering them excellent candidates for transcatheter aortic valve implantation (TAVI). 


\section{Case presentation}

A 76-year-old female affected by FD and AS was referred to our center in order to be evaluated for TAVI. To the best of our knowledge no other similar cases have been reported in the literature to date. The clinical background includes NYHA class III heart failure, systemic hypertension, restrictive lung disease with pulmonary hypertension, previous cerebro-vascular accident, peripheral vascular disease, acroparesthesias and stage 3 chronic kidney disease (baseline creatinine clearance of $37.6 \mathrm{ml} / \mathrm{min}$ ). Diagnostic coronary angiography reported no significant coronary artery disease. In view of multiple comorbidities including a significantly impaired left ventricular ejection fraction (LVEF=21\%), the patient was deemed inoperable by cardiac surgeons (logistic EuroSCORE of 59.1 and a STS Score of 5.5). Baseline ECG demonstrated LV hypertrophy and a prolonged PR interval. Echocardiography showed severe aortic stenosis with mean gradient of $31 \mathrm{mmHg}$, aortic valve area of $0.4 \mathrm{~cm}^{2}$, associated moderate aortic regurgitation and left ventricular hypertrophy (interventricular septum in diastole, IVSd, measured at $14 \mathrm{~mm}$ ) with a restrictive filling pattern. Low-dose dobutamine stress echocardiography $(5-10 \mu \mathrm{g} / \mathrm{kg} / \mathrm{min})$ showed an improvement of stroke volume and ejection fraction of $>20 \%$ (stroke volume index from 27 to $43 \mathrm{ml} / \mathrm{m}^{2}$ ) and an increase of mean gradient to $>40 \mathrm{mmHg}$ (from 31 to $53 \mathrm{mmHg}$ ) defining a state of "true severe" AS, indicating moderately preserved LV contractile reserve. Pre-operative computed tomography findings are shown in Figure 1.

Considering the clinical scenario of "true severe" low-flow low-gradient AS in which the primary culprit of symptoms was deemed to be the valve disease (while LV dysfunction was considered to be a concomitant phenomenon) in a patient with associated high-surgical risk status (as defined by risk scores and other comorbidities) we opted for TAVI.

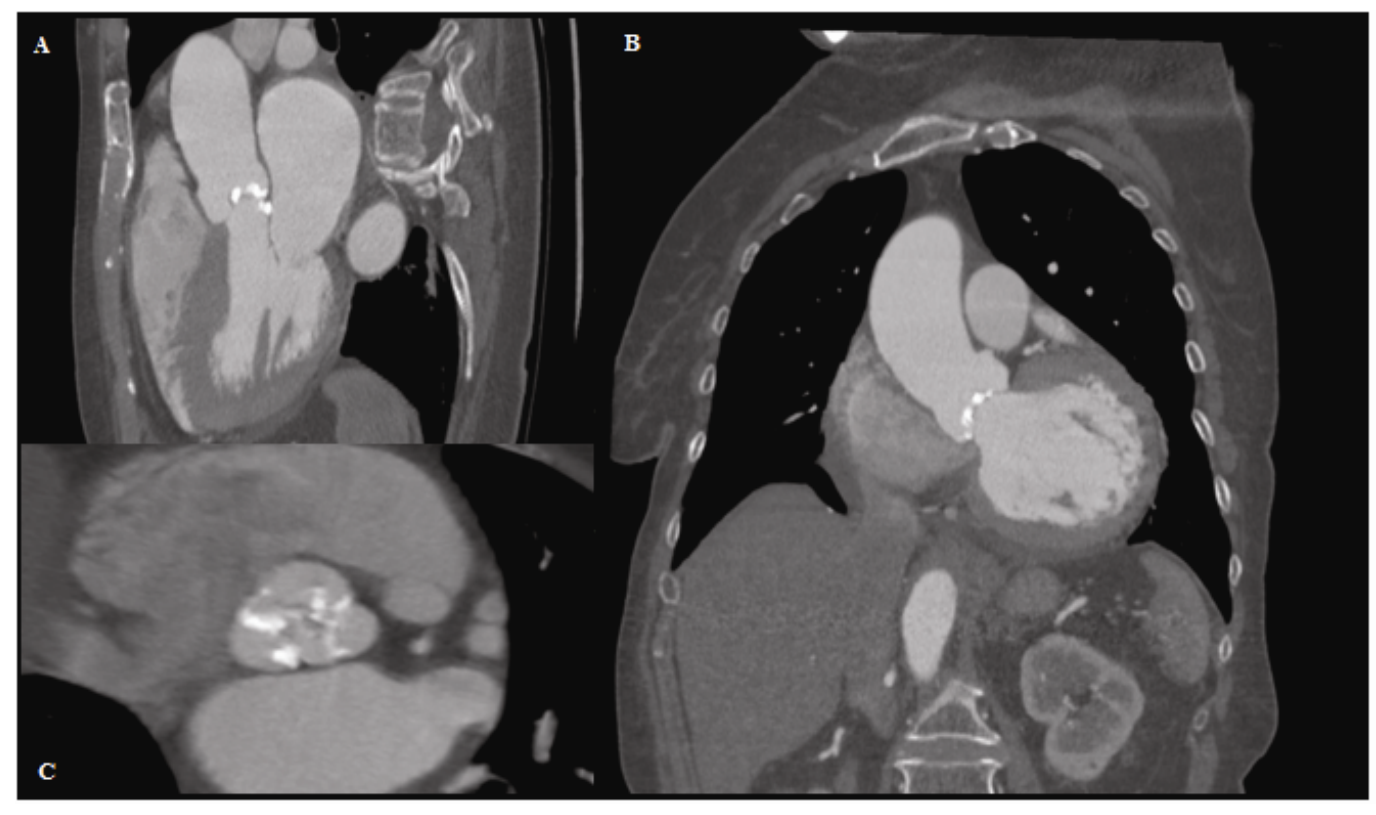

Figure 1. Pre-operative computed tomography scan in a patient affected by Fabry's disease and severe aortic stenosis. (A, $\mathrm{B}$ and C) Cardiac computed tomography showing an aortic annulus of $23 \mathrm{~mm} \times 21 \mathrm{~mm}$, mild leaflet calcifications and a mildly dilated ascending aorta of $40 \mathrm{~mm} \times 41 \mathrm{~mm}$. Other findings were interventricular septal hypertrophy, basal lung fibrosis and bilateral kidney cortical cysts.

A SAPIEN XT Valve $26 \mathrm{~mm}$ was implanted under conscious sedation using a transfemoral approach (see Figure 2). No complications occurred during the procedure or in the peri-procedural period. The patient was discharged 5 days later. Her pre-discharge echocardiogram revealed a marked improvement of the LVEF to $41 \%$, a mean prosthesis gradient of 8 $\mathrm{mmHg}$ and only mild paravalvular regurgitation $(1+/ 3+)$. At 1 month follow-up her clinical condition improved further (NYHA class II) and repeat echocardiogram showed sustained left ventricular systolic function (LVEF of 40\%). A year 
later, her LV systolic function improved even further (LVEF of 53\%). Three years after the procedure, the patient is still alive with stable symptoms. No adverse events occurred during the follow-up period.

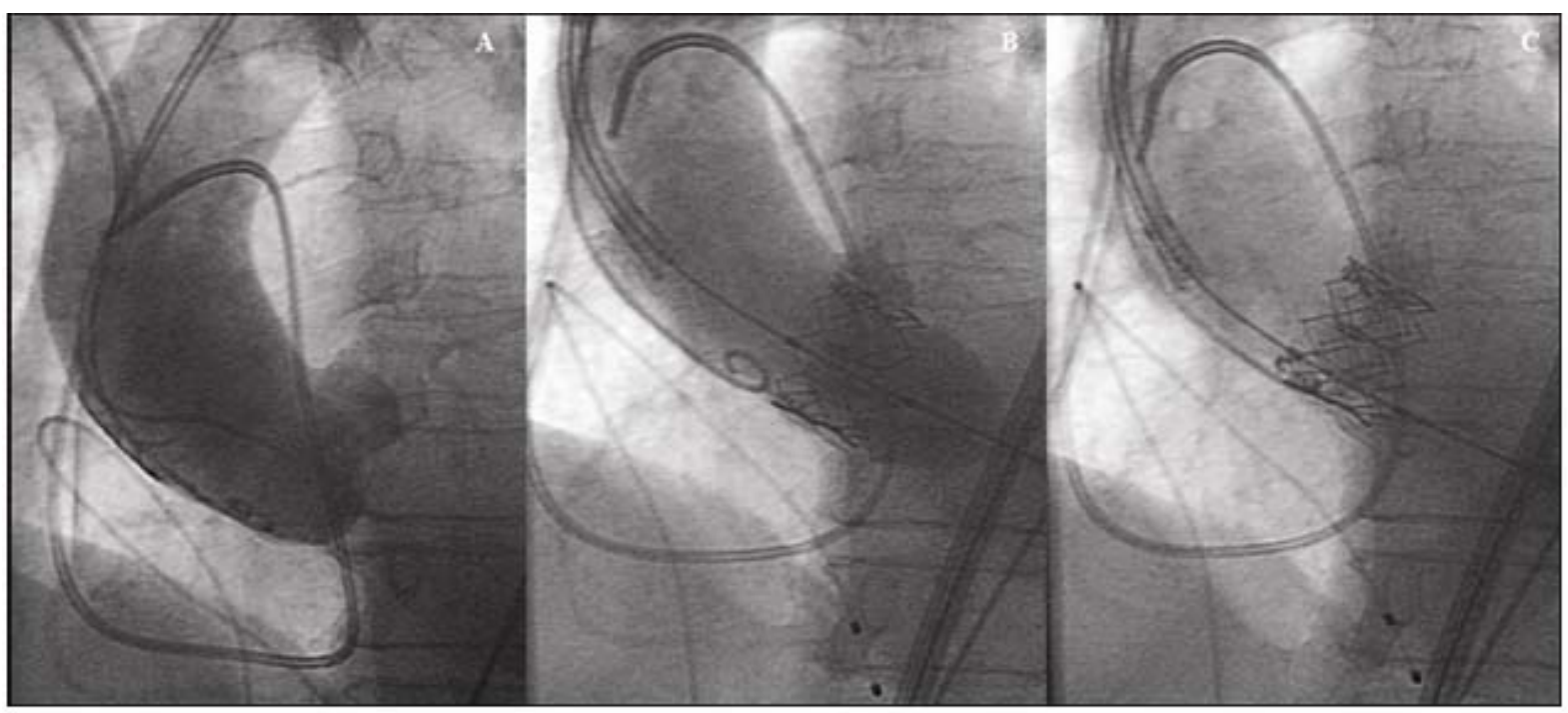

Figure 2. TAVI in a patient affected by Fabry's disease and severe aortic stenosis (fluoroscopic views). (A) Pre-implantation aortography. (B) Correct implantation of the $26 \mathrm{~mm}$ balloon-expandable SAPIEN XT Valve. (C) Final position of the aortic valve prosthesis without the need of valvular balloon post-dilation.

\section{Discussion}

Cardiac involvement in FD is common, caused by glycosphingolipid deposition within myocardial and valvular tissue, and manifests with cardiomyopathy, coronary artery disease, arrhythmias and valvular disorders ${ }^{[2]}$. The myocardium of the LV is most commonly involved, leading to concentric hypertrophy and in later stages restrictive fibrotic cardiomyopathy with systolic and diastolic dysfunction, higher diastolic filling pressures and depressed LVEF ${ }^{[4]}$. In addition, heart valves can also be involved in the pathologic process, in a spectrum ranging from regurgitation, prolapse or valve stenosis ${ }^{[3]}$. As reported in the literature, the presence of LV involvement, alongside coexistent valvular disease, indicate the severity of cardiac involvement ${ }^{[4]}$. Valvular changes in FD relate to glycosphingolipid deposition and fibrosis, causing secondary valvular leaflet stiffening and / or retraction ${ }^{[3]}$. Obviously, the involvement of the aortic valve in the disease process in a patient with an already compromised LV can lead promptly to haemodynamic and clinical deterioration. Reduction of the LV outflow obstruction, can lead to a decrease in LV diastolic filling pressures, improvement of cardiac output and subsequently clinical symptoms.

This patient had "true severe" low-flow low-gradient AS defined by the presence of LVEF $<40 \%$, mean gradient $<40$ $\mathrm{mmHg}, \mathrm{AVA}<1.0 \mathrm{~cm}^{2}$ and increase of stroke volume $>20 \%$ and mean gradient to $>40 \mathrm{mmHg}$ after low-dose dobutamine stress test. Patients with "true severe" type low-flow low-gradient AS generally benefit from aortic valve replacement, whereas patients with the "pseudo-severe" type may not, stressing the paramount importance of a comprehensive preoperative work-up. Even though surgical aortic valve replacement (SAVR) remains the gold standard therapy for severe AS ${ }^{[5]}$, in the current TAVI era, such type of patients (frail and affected by multiple comorbidities) can be successfully treated with a percutaneous approach. As demonstrated by a substudy of the PARTNER trial, that analysed the impact and outcomes of therapy (medical management, SAVR or TAVI) in low-flow low-gradient AS, inoperable patients with this condition treated with TAVI had improved survival as compared to the medical management group while high-risk patients treated with TAVI had similar outcomes to patients who underwent SAVR ${ }^{[6]}$. Indeed, impaired LVEF $(\leq 35 \%)$ does not have a negative impact on TAVI outcomes ${ }^{[7]}$, neither predicts adverse outcomes at 30-day and long-term follow-up. Of note, improvement of LVEF has been reported to be higher in patients with impaired LVEF, as 
compared to patients with normal pre-procedural LV function (LVEF $>35 \%$ ) ${ }^{[8]}$. Furthermore, as recently reported in a substudy of the cohort A of the PARTNER trial ${ }^{[9]}$, in patients with LV dysfunction and high-surgical risk, mortality rates and LV functional recovery are comparable between TAVI and SAVR.

Finally, as demonstrated in the current case, despite the presence of a severe clinical condition involving multiple organs, treatment of the valvular disorder led to a great improvement of LVEF (from $21 \%$ to $53 \%$ after 1 year) and clinical symptoms. It seems therefore that TAVI is a viable therapeutic option in severe AS patients with impaired left ventricular function and an underlying systemic disorder.

\section{Conclusion}

This is the first case reported in literature suggesting that TAVI procedure is an effective therapeutic option in patients with FD and associated severe AS. Depressed LVEF should not be considered a contraindication to TAVI procedure, even in the presence of a severe underlying cardiomyopathy such as the one caused by Fabry's disease.

\section{References}

[1] Germain DP Fabry disease. Orphanet J Rare Dis. 2010; 5: 30. http://dx.doi.org/10.1186/1750-1172-5-30

[2] Morrissey RP, Philip K.J, Schwarz ER Cardiac abnormalities in Anderson-Fabry disease and Fabry's cardiomyopathy. Cardiovasc J Afr. 2011; 22(1): 38-44.

[3] Desnick RJ, Blieden LC, Sharp HL, Hofschire PJ, and Moller JH. Cardiac valvular anomalies in Fabry disease. Clinical, morphologic, and biochemical studies. Circulation. 1976; 54: 818-25. http://dx.doi.org/10.1161/01.CIR.54.5.818

[4] Kampmann C, Baehner F, Ries M, Beck M. Cardiac involvement in Anderson-Fabry disease. Journal of the American Society of Nephrology : JASN. 2002; 13 Suppl 2: S147-9.

[5] Nishimura RA, Carabello BA, Faxon DP et al. ACC/AHA Guideline update on valvular heart disease: focused update on infective endocarditis: a report of the American College of Cardiology/American Heart Association Task Force on Practice Guidelines endorsed by the Society of Cardiovascular Anesthesiologists, Society for Cardiovascular Angiography and Interventions, and Society of Thoracic Surgeons. Journal of the American College of Cardiology. 2008; 52: 676-85. http://dx.doi.org/10.1016/j.jacc.2008.05.008

[6] Herrmann HC, Pibarot P, Hueter I et al. Predictors of mortality and outcomes of therapy in low-flow severe aortic stenosis: a Placement of Aortic Transcatheter Valves (PARTNER) trial analysis. Circulation. 2013; 127: 2316-26. http://dx.doi.org/10.1161/CIRCULATIONAHA.112.001290

[7] van der Boon RM, Nuis RJ, Van Mieghem NM et al. Clinical outcome following Transcatheter Aortic Valve Implantation in patients with impaired left ventricular systolic function. Catheterization and cardiovascular interventions : official journal of the Society for Cardiac Angiography \& Interventions. 2012; 79: 702-10. http://dx.doi.org/10.1002/ccd.23423

[8] Fraccaro C, Al-Lamee R, Tarantini G et al. Transcatheter aortic valve implantation in patients with severe left ventricular dysfunction: immediate and mid-term results, a multicenter study. Circulation Cardiovascular interventions. 2012; 5: 253-60. http://dx.doi.org/10.1161/CIRCINTERVENTIONS.111.964213

[9] Elmariah S, Palacios IF, McAndrew T et al. Outcomes of Transcatheter and Surgical Aortic Valve Replacement in High-Risk Patients With Aortic Stenosis and Left Ventricular Dysfunction: Results From the Placement of Aortic Transcatheter Valves (PARTNER) Trial (Cohort A). Circulation Cardiovascular interventions. 2013; 6: 604-14. http://dx.doi.org/10.1161/CIRCINTERVENTIONS.113.000650 